\title{
Historia de la locución radiofónica en Colombia: caracterización de unas posibles etapas ${ }^{1}$
}

\author{
Óscar Julián Cuesta Moreno ${ }^{2}$
}

\begin{abstract}
Resumen
El artículo presenta los resultados de una investigación que tuvo como objetivo dar cuenta de las características de la historia de la locución radiofónica en Colombia. Para ello, se realizó en primer lugar una búsqueda documental en esta línea; en un segundo momento, se efectuaron entrevistas en profundidad a cinco locutores de más de 35 años de experiencia en esta labor. El texto propone, como conclusión, la formulación de cuatro etapas históricas para la locución colombiana y establece la relación de estos momentos con el desarrollo tecnológico en la radiodifusión.
\end{abstract}

Palabras clave: Locución, radio, producción radial, tipos de locución y locutores colombianos.

1 Este artículo es producto del proyecto de investigación Por una historia de la locución radiofónica colombiana. Desarrollado en el año 2011 por el Grupo de Investigación Comunicación, Cultura y Tecnología, de la Fundación Universitaria Los Libertadores.

2 Comunicador Social de la Universidad Santo Tomás, Magister en educación de la Universidad Pedagógica Nacional, especialista en docencia universitaria de la Universidad Cooperativa de Colombia, y Técnico en locución y producción de medios audiovisuales. Docente de la Fundación Universitaria Los Libertadores. Correo: oscarcuesta@colombia.com 


\title{
History of Colombia radio broadcasting: characterization of possible stages
}

\begin{abstract}
This article presents the results of a research study to account for the characteristics of radio broadcasting history in Colombia. To do this, first a documentary inquiry on this line was conducted, then in-depth interviews were made to five speakers with over 40-year-experience in this field. The text advances, as a conclusion, four historical stages in Colombian radio broadcasting narration and establishes the relationships between those milestones and technological developments in radio broadcasting.
\end{abstract}

Key words: Narration, radio, radio broadcasting, types of narration.

Recibido: 09 de marzo 2012

Aceptado: 17 de mayo 2012

\section{Introducción}

La radio ha jugado un importante papel en el proyecto de construir una nación colombiana, pues sus cualidades técnicas permitieron difundir unos mismos referentes sociales para un país fragmentado en diversas regiones por una accidentada orografía. Así, por ejemplo, la transmisión de la vuelta a Colombia en bicicleta a mediados del siglo XX permitió que un país fraccionado cultural y geográficamente siguiera el reto deportivo por las abruptas carreteras nacionales.

Consecuentemente, las voces de las personas que socializaban los mensajes a través del medio radial se convertían en íconos de las producciones que informaban, educaban y entretenían a la población. En ese orden de ideas, el locutor se instauró en la dinámica nacional de una manera preponderante, en donde el cultivo de la voz, el buen uso del lenguaje y el conocimiento sobre datos de la cultura general eran condiciones intrínsecas.

El presente artículo, precisamente, presenta los resultados de una investigación que pretendió caracterizar la historia de la locución radiofónica en Colombia, 
procurando establecer unas posibles etapas del desarrollo de esta profesión desde su origen hasta hoy.

Es pertinente decir que, en primer lugar, no existen mayores antecedes sobre este tema, así que la investigación es un primer esfuerzo por dar cuenta de la historia de esta profesión en el país. Como antecedentes tangenciales se pueden citar dos artículos sobre la historia de la Asociación Colombiana de Locutores y Comunicadores (ACL), uno de ellos en la página web de la misma asociación y otro en la enciclopedia libre Wikipedia.

Otros trabajos sobre la locución colombiana son el de Romero (1947) escribió un artículo de exhortación para los locutores de la época. En 1985 el Ministerio de Comunicaciones publicó una cartilla para locutores que expresaba las condiciones legales de la profesión y, además, describía las cualidades intelectuales, léxicas y de técnica vocal para ejercer este oficio. Buitrago (1993) realiza un análisis de la credibilidad de los locutores y periodistas frente a los oyentes. Siguiendo otras inquietudes, Landázuri (2008) presenta una indagación con locutores miembros de la ACL en la ciudad de Bogotá acerca de la prevención vocal en el marco de la fonoaudiología.

Si bien existen varios libros sobre entrenamiento para el locutor y los diferentes tipos de locuciones, la investigación no consideró pertinente abordarlos en sus marcos referenciales, pues desbordaban el interés de las categorías centrales de análisis y el propósito de caracterizar la historia de la locución colombiana.

Se puede afirmar, pues, que el trabajo presentado en este artículo se convierte en un abordaje novedoso para la materia dadas las pocas referencias en esta línea. Ese vacío, por ende, señala la pertinencia de este trabajo, dado que el papel del locutor radiofónico tiene una intrínseca relación con la historia de la radio y con las dinámicas sociales del país.

\section{Metodología}

Para caracterizar la historia de la locución colombiana y plantear las posibles etapas de su secuencia histórica, la investigación siguió un enfoque cualitativo de corte interpretativo. Las técnicas utilizadas fueron las siguientes: en un primer momento, la revisión documental de las publicaciones sobre locución colombiana y sobre las leyes y decretos que han sentado alguna jurisprudencia en el ejercicio de este oficio.

En una segunda etapa, se realizaron entrevistas en profundidad con cuestionarios semi-estructurados a cinco locutores de reconocida trascendencia nacional. Como primer ítem los locutores debían ser colombianos; en segundo lugar, de preferencia tener más de 35 años de experiencia como locutor, sin importar su ejercicio 
(locutor de noticias, deportivo, musical, etc.); como tercer criterio, se buscaron locutores que siguieran ejerciendo en la actualidad, para que los entrevistados tuvieran mayores criterios de análisis al momento de hacer parangones históricos.

Los cinco locutores fueron: Ernesto Rojas Ochoa (50 años de experiencia), actual Director de Programación de la Cadena Colmundo Radio y formador de locutores en el Colegio Superior de Telecomunicaciones; Gustavo Niño Mendoza (38 años de experiencia), fue locutor de noticias de Caracol y RCN, hoy trabaja en la cadena Melodía de Colombia; Jorge Eliecer Campusano (47 años de experiencia), locutor deportivo de Radio Sutatensa, Todelar, Caracol y RCN; Fred Emiro Núñez (38 años de experiencia), narró más de 300 documentales de Colciencias y 400 documentales de National Geographic con RTI, locutor de noticias y actualmente voz comercial de transmisiones de fútbol; Gabriel Muñoz López (62 años de experiencia), narrador deportivo, comentarista y musicólogo, en la actualidad conduce el programa Así Canta Colombia de Caracol Radio.

En una tercera etapa se realizaron análisis a 20 archivos de audio de transmisiones radiofónicas colombianas. El archivo no se limitó a ninguna emisora o programa específico, sino que se seleccionaron archivos de manera aleatoria para no limitar la muestra a gustos particulares de los investigadores. El propósito de revisar los archivos fue determinar características en la locución según el contexto histórico de emisión, para aumentar el número de criterios para esbozar unas posibles etapas de la historia de la locución.

\section{Resultados y discusión}

En primer lugar, las entrevistas y los análisis realizados permiten identificar que una posible clasificación de las etapas de la locución radiofónica en Colombia es la siguiente:

Locución primigenia de la primera radio (1929-1935)

Contratación de locutores y locución de rasgos informativos (1935-1948)

Locución deportiva y de radio novelas (1948-1975)

Locución de emisoras juveniles, nuevo estilo informativo e informal (1975-hasta hoy)

A continuación se procuran desarrollar las características de estas etapas. Los atributos para cada etapa están basados, básicamente, en lo expuesto por los locutores en las entrevistas y el análisis a los archivos escuchados. 
Si bien la radio inicia en Colombia en 1923 con radioaficionados que importaron los primeros transmisores-receptores, el nacimiento oficial de la radiodifusión en el país se da en 1929, con la inauguración de la HJN, más tarde Radiodifusora Nacional, en el gobierno de Miguel Abadía Méndez. Este año, igualmente, se funda la primera estación privada, la $\mathrm{HKD}$, conocida como la Voz de Barranquilla (Pareja, 1984).

Dado que en los primeros años el medio estaba producido por personas de las clases altas socioeconómicas aficionadas a la radiodifusión y, por el alto costo de los receptores, era igualmente sintonizada por personas de este mismo estrato, los primeros programas eran de "música clásica, música de cámara, ópera, zarzuela y las primeras adaptaciones del teatro español (...) Los programas complementarios eran también de tipo cultural tales como la poesía, los comentarios de literatura, las conferencias tanto de tipo académico como de carácter religioso" (Pareja, 1984, p. 22).

Teniendo en cuenta lo anterior, se pudo especificar en la investigación que los rasgos característicos de la locución en esta primera etapa respondía a un lenguaje de personas letradas, con vocabulario amplio y sintaxis de escuela española. Sin embargo, el estilo de presentación tenía influencia norteamericana, pues muchos de los radicalistas colombianos conocían el estilo de allí ${ }^{3}$. La voz empezó a tener un rasgo engolado, es decir, procurando cierta resonancia en el fondo de la boca o de la garganta.

Es pertinente destacar que, dado que la mayor parte de la programación era música, más exactamente la música que le gustaba al dueño, la locución de esta primera etapa era de lo que hoy se denomina de presentación musical, que en algunas estaciones llaman DJ.

Como estas primeras emisoras aseguraban su sostenibilidad con canjes publicitarios, se empezó a dar las primeras locuciones publicitarias. No obstante, eran todavía bastante informales y tenía una notable carencia técnica. Estas locuciones publicitarias se basaban en la mención de los patrocinadores.

Es importante referir que con la aparición de la Voz de la Víctor, en 1933, la radio tuvo una mayor popularización, puesto que difundía música típica colombiana y boleros. Sin embargo, el hecho de programar este tipo de música no implica que gran parte de la población tuviera acceso al medio radial.

Contratación de locutores y locución de rasgos informativos (1935-1948)

La radio tuvo un especial crecimiento con el desarrollo económico producto da las iniciativas de industrialización del gobierno de Alfonso López Pumarejo. Aquí se da un importante crecimiento de las pautas publicitarias en las diferentes 
Historia de la locución radiofónica en Colombia: caracterización de unas posibles etapas, artículo producto de la investigación

emisoras, puesto que los industriales encontraron en ellas un buen espacio de socialización. Aún más, muchas industrias fundaron directamente estaciones.

El impulso económico del país influyo de manera consecuente en la producción radial: "El sistema estaba caracterizado por la presencia de una 'hombre orquesta', normalmente el dueño, que realizaba todas las funciones. El resultado era una improvisación constante. Se impuso entonces el criterio de organización empresarial: administración y producción técnica" (Pareja, 1984, p. 29). A esto se sumó la amplitud en los horarios de transmisión, lo que obligó una mayor exigencia en la producción. En ese orden de ideas, se puede afirmar que en este periodo ${ }^{4}$ se empiezan a contratar los locutores, es decir, se le paga a una persona para que hable ante el micrófono para dar avisos, hacer comentarios, brindar información, presentar música, etc.

Dado que, como lo señala Pareja $(1984)^{5}$, en las décadas del treinta y cuarenta se aumenta el número de emisoras, comienza la competencia por la sintonía, es decir, por los patrocinadores. En esta puja los locutores empiezan a tener importante protagonismo, pues según sus rasgos, simpatía y popularidad en los oyentes las emisoras pujaban por contratarlos y así "arrastrar" audiencia.

Por otro lado, este periodo parece dar el origen a la primera locución de rasgos informativos. Esto se da en el marco de una creciente tensión entre la competencia radio y prensa, donde esta última tenía el monopolio noticioso. En un principio, pues, los locutores leían la prensa, dado que esta tenía la infraestructura del servicio noticioso, como era la recepción de los cables internacionales de las agencias AP, Reuter, etc.

Los periódicos de la época lograron prohibir por decreto ${ }^{6}$ la lectura de noticias publicadas en sus páginas: justificaban que al medio escrito le costaba conseguir y publicar información, mientras que la radio lo hacía sin la mayor inversión y sí con gran beneficio.

Se puede afirmar que en 1935 inicia con rasgos formales la locución informativa o de noticias, pues allí se comienzan a establecer los radioperiódicos ${ }^{7}$. Según algunos entrevistados, la muerte de 26 personas, entre ellas Carlos Gardel, en un accidente aéreo el 24 de junio de 1935 en la ciudad de Medellín es un episodio relevante en la locución informativa, pues Antonio Henao Gaviria, de la Voz

\footnotetext{
4 Pareja (1984) en su libro sobre historia de la radio en Colombia clasifica el periodo de 1935-1940 como de lanzamiento y consolidación de la radio comercial, para esta investigación dicho periodo va hacer parte de la Contratación de locutores y locución de rasgos informativos (1935-1948).

5 El autor señala que en 1934 había 17 emisoras, para 1939, 44.

6 Decreto 627 del 23 de marzo de 1934.

7 Gustavo Rodas Isaza en 1935 creó en la Voz de Antioquía el primer radioperiódico del país. Sin embargo, él venía realizando los primeros ejercicios de periodismo radiofónico desde 1932 en la emisora Ecos de la Montaña en Medellín (Muñoz y otros, 2008). Esto hace a Rodas Isaza un protagonista de la locución informativa.
} 
de Antioquía, estaba presente en el aeropuerto, y transmitió telefónicamente el desarrollo de los acontecimientos desde el lugar mismo de la noticia.

El anterior acontecimiento, sin duda, separa la lógica informática del periódico y la radio, pues esta última podía transmitir los hechos desde el lugar y en directo; así, la radio se convirtió en actualidad, en el "última ahora", y los periódicos empezaron hacer el periodismo de análisis y profundización informativa.

En consecuencia, la tensión que impedía que en la radio se leyeran las notas publicadas por los periódicos y las posibilidades técnicas de transmitir en directo los acontecimientos, llevó a que la radio esquematizara sus propios sistemas y estilos de información, lo cual influenció directamente en la consolidación de la locución informativa y sus características. No hay que olvidar, además, que esta locución informativa estuvo influenciada por los intereses políticos propios de la época:

Teniendo en cuenta que las luchas políticas de Colombia, especialmente las luchas bipartidistas entre liberales y conservadores marcaron por varias décadas la historia colombiana, los espacios informativos radiales se convertían en medios de difusión, propagación, controversia, debate y oposición a las ideas políticas que luchaban por el poder (Muñoz y otros, 2008, p. 57).

En 1948, con el asesinato de Jorge Eliecer Gaitán, esas manifestaciones políticas se hicieran más que explícitas en muchos locutores. Incluso, algunos de ellos incentivaron la revuelta popular a través de los micrófonos que dio pie al Bogotazo. Después de estos episodios muchas licencias fueron canceladas y para regularlas se creó la Asociación Nacional de Radiodifusión, las estaciones para poder transmitir debían estar afiliadas.

Los avisos publicitarios para este periodo respondían al auge de la creciente industria interna, en consecuencia, la locución publicitaria promocionaba cerveza, vidrios, zapatos, fósforos, café, textiles, jabones, entre otros.

Locución deportiva y de radio novelas (1948-1975)

Pareja (1984), en su historia de la radiodifusión en Colombia, denominó el periodo de 1948 y 1957 como la etapa de la consolidación de las cadenas. En la investigación realizada se propone denominar el periodo de 1948 a 1975 como el de la locución deportiva y de radionovelas, géneros que se desarrollaron, entre otras cosas, gracias al afianzamiento de las cadenas radiales estipuladas por Pareja, pues los enlaces que dan origen al encadenamiento tuvieron como gran eslabón las transmisiones, por ejemplo, de ciclismo y fútbol. 
Historia de la locución radiofónica en Colombia: caracterización de unas posibles etapas, artículo producto de la investigación

Las radionovelas $^{8}$ y radioteatros ${ }^{9}$ tuvieron una importante fortificación en este periodo, pues "Los empresarios comenzaron a demostrar preferencia por las emisoras que utilizaban este género (...) se dieron cuenta que había una audiencia atenta" (Pareja, 1984, p. 46). Es pertinente decir que muchos de los actores eran, igualmente, locutores.

En esta etapa se da un hecho fundamental para la locución: el 23 de agosto de 1949 se establece el decreto 2574, por el cual se reglamenta la expedición de licencias de locutores o radiofonistas. Estas licencias las otorgaba el Ministerio de Correos y Telégrafos a los locutores que demostraban sus capacidades en un examen ${ }^{10}$ realizado por la Asociación Nacional de Radiodifusión.

En estos exámenes "Los locutores eran calificados en virtud de su voz, sin duda alguna, y en virtud de su educación general" (Pareja, 1984, p. 75). Estas cualidades de la voz tenían, inevitablemente, componentes innatos o naturales: la tonalidad y la buena disposición de los órganos articuladores. Sin embargo, también dependía de variables formativas como la respiración, la buena dicción (articulación correcta de sonidos consonánticos), la vocalización (articulación correcta de sonidos vocálicos) y la cadencia en la lectura. Es de ponderar que las pruebas también evaluaban los conocimientos de cultura general y de gramática castellana, es decir, que el locutor no solamente limitaba su capacidad a la voz, sino al bueno uso de ésta en la comunicación como un proceso más complejo. Siguiendo la línea de la normatividad, hay que decir que para después de 1948 se expiden varias reglamentaciones que controlan la emisión de los mensajes radiofónicos, lo que consecuentemente afecta a los locutores. Entre otros, se pueden citar: el decreto 0997 de 1951, que aumenta la pena de prisión para quienes operaran radioemisoras de manera clandestina; el decreto 3418 de 1954, que regula las telecomunicaciones pues establece que estas son propiedad del Estado (viajan por el espectro electromagnético), pero que él las puede ceder a personas naturales o jurídicas. El artículo 8 de este decreto prohíbe explícitamente los comentarios y que el gobierno reglamentará la manera como se transmite la información y las exposiciones radiales. Precisamente, el decreto 2427 de 1956 establece esta reglamentación.

Según Pareja (1984), los controles ejercidos llevaron a que los contenidos de las emisoras buscaran temáticas superficiales o menos comprometedoras, incrementando el número de radionovelas y transmisiones deportivas. Si bien hay otras coyunturas que explican el incremento de este tipo de programas, como por ejemplo el nacimiento del fútbol profesional colombiano (se empezó a jugar desde 1948) y de la vuelta a Colombia en bicicleta $(1951)^{11}$, lo cierto

\footnotetext{
8 Sin embargo, es importante recordar que la aparición de las novelas en la radio tiene su antecedente en la emisión de Yon-fu, en 1938 (Pareja, 1984).

9 El radioteatro adaptaba una obra de teatro en una radiodramatización.

10 Las características de la prueba están explicitadas en la Decreto 1051 del 29 abril de 1944
} 
es que en estas décadas la locución para estos tipos de contenidos cobra significativa relevancia, de allí que la investigación se permita proponer y denominar esta etapa de dicha manera. ${ }^{12}$

Por otra parte, aunque no es de notoria relevancia cuantitativa pero sí cualitativa, en este periodo nace la locución cultural. Caso emblemático es el de la HJCK 'El mundo en Bogotá', de Álvaro Castaño Castillo, que desde 1950 impuso un estilo paradigmal para los locutores culturales. Del mismo modo, se podría hablar del nacimiento de la locución de propósitos educativos con el nacimiento de la emisora Radio Sutatenza, que acompañaba las lecciones dictadas radiofónicamente con la presencia de monitores presenciales y cartillas impresas.

\section{Locución de emisoras juveniles, nuevo estilo informativo e informal}

Las emisoras juveniles comenzaron a mediados de la década de los 60'. $\mathrm{Su}$ formato, en líneas generales, era la presentación musical, pero su innovación fue la de ofrecer música que representaba el sentido de las nuevas generaciones y que permitía expresar las identidades renovadas y florecientes, como por ejemplo el rock and roll de la estación Radio Quince. Como consecuencia, se formaliza el rol del DJ' o disc jockey, es decir, el encargado de seleccionar, presentar y poner la música producida por un artista. Para la época, se destacaron DJ's como Carlos Pinzón y Alfonso Lizarazo (Vargas, 2009).

Más adelante, en la década del 80, se populariza el formato juvenil matutino, cuyo ejemplo más representativo es el Zoológico de la Superestación, 88.9. Este programa marcó un hito en la radio juvenil que influyó en las producciones de las otras emisoras. El formato matutino de las radio-estaciones juveniles tuvo, sin lugar a dudas, una innegable influencia del modelo Howard Stern ${ }^{13}$, es decir, contenidos cargados de humor, lenguaje de doble sentido y sarcasmo. Este formato se mantiene hoy en día y es fuente permanente de crítica. De hecho, los locutores entrevistados fueron reiterados en censurar la labor del actual locutor juvenil, pues ha perdido las cualidades que otrora hicieron famosos a los locutores colombianos: conocimientos amplios de la cultura general, gusto por la literatura y la poesía y un rico y estricto manejo del vocabulario, la gramática y la pronunciación.

\footnotetext{
11 Hay que decir, no obstante, que varios autores asociación el nacimiento de estos dos certámenes deportivos a intereses gubernamentales que buscaban distraer a una nación sumida en una guerra partidista y fratricida.

12 Es importante decir que el aumento en contenidos deportivos y, por extensión, el incremento del protagonismo de la locución deportiva se debió, igualmente, a las transmisiones de eventos internacionales, como las Olimpiadas y los Panamericanos. Además, es de recordar que muchos llaman a Carlos Arturo Rueda C. como el padre de la locución deportiva en el país.

13 Haward Stern es un locutor que renovó la radio norteamericana. Sus programas, muchas veces autocensurados por las mismas cadenas donde él trabajaba, tenían una audiencia masiva. Desde hace unos lustros, cuando dejó de laboral en la reconocida NBC, se convirtió en uno de los pioneros de la emisión web. Su vida quedó retratada en la película autobiográfica Partes Privadas.
} 
Por su parte, la locución informativa sufrió una renovación con los nuevos formatos noticiosos. En los 80 desaparece el radioperiódico y se pasó a una mistura de noticias entrevistas, crónicas y radioreportajes (Muñoz y otros, 2008, p. 58). Uno de los artífices de estos cambios fue el estilo impuesto por Yamit Amat cuando asumió la dirección de Caracol Radio. Amat, entre otras cosas, puso corresponsales en las capitales colombianas y en las principales ciudades del mundo. Así, los noticieros dejaron de ser lecturas simples de noticias, para utilizar con más protagonismo el reporte directo de la información por el mismo periodista, la entrevista al involucrado en el hecho noticioso y los reportajes radiales.

Como se puede ver, en la radio informativa fue cada vez más notorio el rol del periodista, y el locutor tuvo una labor más secundaria. El papel del locutor migró al estilo anchorman: dice la generalidad de la noticia y da paso al periodista que extiende la información. Es un estilo menos formal, donde el titular no tiene una lectura rígida ( anchorman comenta la información y pregunta y contra-pregunta al periodista (Salgado, 2005). Es el formato vigente en los noticieros matutinos en las grandes emisoras colombianas y ya no son los locutores los que realizan el papel de 'hombre ancla' ${ }^{14}$, sino el conductor del noticiero, por lo general el mismo director. No obstante, la locución tradicional de lectura de noticias aún se puede escuchar en ciertas emisiones (como el estilo del médico Cristóbal Américo Rivera en La Cariñosa de RCN o el de Gustavo Niño Mendoza en la Cadena Melodía).

Dado que hoy la radio tiene más posibilidades gracias a su marco legal (Decreto 2805 DE 2008) $)^{15}$, que permite la existencia de emisoras comunitarias, de interés público y comerciales, la locución tiene una carácter menos formal al que se pudo escuchar en las primeras etapas. Si bien existen más escuelas de formación para comunicadores y academias para locutores, no todas las personas que hablan por los micrófonos de una emisora han realizado cursos de preparación. En efecto, los locutores entrevistados afirmaron la necesidad de revivir la licencia del locutor y garantizar así que los radio hablantes tengan unas competencias mínimas comprobadas frente a un evaluador.

Por otro lado, hablar de la locución en la dinámica radial contemporánea implica reconocer que los avances tecnológicos en la producción radial han modificado el estilo del locutor. De hecho, se corrigen errores o se colorea la voz de manera digital con diferentes software, cuando antes implicaba un proceso denodado de puesta en escena del locutor. Además, la posibilidad que brindan los nuevos entornos digitales y de inter-conectividad en la configuración de nuevos oyentes, expone a los locutores a una interacción permanente, casi íntima, con sus

14 Anchor, ancla y man, hombre.

15 Es necesario decir que este marco no tiene en cuenta las emisoras vía web, que hoy son una oferta significativa. 
radioescuchas. Esto obliga a los presentes y futuros locutores a repensar el uso o desuso de las posibilidades multimediales e hipertextuales de su labor en la nueva radio. Con respecto a la voz, en esta última etapa ya no se buscan tonalidades graves ni se imposta la pronunciación como las décadas pasadas, se privilegia más la naturalidad, pero igualmente se exige la correcta articulación de las palabras.

Si bien la locución de doblaje no es propia de la radio y su escenario inmanente es la televisión o el cine, es pertinente destacar el papel de los locutores colombianos en esta labor, dado que su labor es bien cotizada en el mercado latinoamericano, en especial, por su acento más neutro y su español más universal.

\section{La formación de locutores}

Si bien la investigación no tuvo como eje analizar la formación de locutores, es necesario decir que las consultas realizadas permiten afirmar que las escuelas o instituciones que tienen programas en esta línea son fundamentales. Los entrevistados dieron cuenta de la formación empírica en muchos de ellos y destacaron el papel de Manual Cabral Jiménez en dicha formación.

Hay que destacar, igualmente, dos centros educativos en esta línea: el Colegio Superior de Telecomunicaciones y la Academia Arco. Sin embargo, después de quedar derogada la licencia de locutor, estas academias sufrieron una disminución significativa en el número de matriculados. Por su parte, en los programas de pregrado de las facultades de comunicación no se observa con gran énfasis componentes que procuren el desarrollo de la locución.

Cabe recordar aquí el contra sentido planteado por Gabriel Muñoz López en su entrevista: los locutores de antaño, sin escuelas de comunicación formales, tenían una mayor preocupación por el idioma, la historia, la cultura general. Hoy, que existen muchas facultades de comunicación social, los profesionales tienen falencias en estas categorías.

\section{Conclusiones}

La investigación pudo determinar que unas posibles etapas que caracterizan la historia de la locución en Colombia son: Locución primigenia de la primera radio (1929-1935); Contratación de locutores y locución de rasgos informativos (19351948); Locución deportiva y de radio novelas (1948-1975); Locución de emisoras juveniles, nuevo estilo informativo e informal (1975-hasta hoy). No obstante, estos cortes temporales no son taxativos y están abiertos a la discusión.

Estas etapas permiten observar que la labor del locutor ha estado afectada por las dinámicas económicas, políticas y sociales del país. De igual manera, se 
puede afirmar que la locución es una actividad que se ha modificado por las dinámicas tecnológicas en la producción radiofónica (de lector de periódicos a contertulio de sus oyentes por redes sociales, se podría decir gráficamente). En el calendario litúrgico católico, el 24 de marzo se conmemora el anunció del Arcángel Gabriel le hizo a María: que ella sería la madre de Jesús. Este día, de carácter informativo, fue tomado para celebrar el día del locutor, lo que muestra la importancia de esta labor en un contexto tradicionalmente católico como el colombiano. Sin embargo, la dignidad que otrora tuvo el locutor ha perdido protagonismo, sobre todo por las nuevas dinámicas radiales que privilegian la informalidad.

Esta informalidad es consecuencia, entre otras cosas, de las nuevas dinámicas de información que privilegian lo comercial y lo ligero, pero principalmente a la derogación de la licencia de locutor. En efecto, cuando un locutor iniciaba su labor, decía el número de su licencia al aire y se identificaba por su nombre, lo que implicaba demostrar públicamente su permiso para ejercer la profesión $\mathrm{y}$, de otro lado, poderle hacer seguimiento a su labor. Sin licencia, creen los entrevistados, bajó la calidad de los profesionales de la radio, pues cualquier puede hablar por un micrófono.

Cabe citar a Jorge Eliecer Campusano: "tener una licencia de locutor, que obtenía uno después de presentarse ante un jurado, lo obligaba a uno a preparase y a tener conocimientos y conciencia del deber que uno adquiría". Además, según Ernesto Rojas, retirar la licencia "abarató la mano de obra", pues las emisoras pueden contratar a cualquier persona, antes sólo a los que tenía certificado legal.

Desde el Decreto 1051 del 29 abril de 1944 se estipuló una prueba para las acceder a las licencias. En ese entonces, se evaluaba la lectura en voz alta (dicción, vocalización, armonía), la gramática castellana, la facilidad de expresión y conocimientos de geografía e historia.

Los requisitos legales para la licencia de locutor sufrieron ciertas modificaciones con los decretos Decreto 2427 de 1956, modificado por el Decreto 356 de 1974, que estipulaba 6 categorías de licencias. Posteriormente, el Decreto 2387 de1983 especifica dos tipos de licencias: para radio y televisión. El último decreto en esta línea fue el Decreto 651 de 1988, que especifica un examen más riguroso para expedir la licencia. ¿Qué tanto se enriquecería la radio con una prueba similar hoy en día? Es una pregunta que admite diferentes hipótesis. Lo que abre espacio para nuevas investigaciones en la línea.

La anterior jurisprudencia quedó anulada con sentencias de la Corte Constitucional que argumentan que las licencias vulneran artículos de la carta magna, principalmente, el artículo 20, que hablan de la libertad de expresión, de informar y recibir información. Sin embargo, en los últimos años se han 
presentado proyectos de ley para recuperar la licencia de locutor y reactivar su carácter profesional (se destaca el Proyecto de ley 228 de 2007 cursado en el Senado y el 109 de 2006 tramitado en la Cámara de Representantes). Hay que decir que estas iniciativas han contado con el acompañamiento de la Asociación Colombiana de Locutores.

Finalmente, se puede decir que los locutores han tenido un papel relevante en la constitución de imaginarios sociales, especialmente, en lo referente a los sentidos de identidad en el marco del proyecto de nación. Esto se puede ver, principalmente, en las transmisiones deportivas: un país fragmentado culturalmente por las regiones geográficas y las prácticas sociales se une siguiendo las emocionantes narraciones de los que pedalazo a pedalazo escalan las montañas europeas, anotan un gol o logran el podio olímpico.

\section{Referencias Bibliográficas}

Asociación Colombiana de Locutores. (S.f). Historia. Recuperado de http://www. vocesacl.com/historia.html el 5 de junio de 2011.

Landazuri, E. (2008). Prevención vocal una responsabilidad fonoaudiológica en los profesionales de la voz. En: Umbral Científico. No. 12. Pp. 33-51

Muñoz, J. y otros. (2008). Evolución histórica del radioperiodismo en Antioquía. En: Revista Lasallista de Investigación. Vol. 5 No. 1. Pp 51-60

Muñoz, J. y otros. (2008). Evolución histórica del radioperiodismo en Antioquía. En: Revista Lasallista de Investigación. Vol. 5. No. 1. Pp. 51-60

Nullvalue. (1990, 31 de diciembre). Yamid Amat se Retira de Caracol. El tiempo.

Pareja, R. Historia de la radio en Colombia 1929-1980. Bogotá: Servicio Colombiano de Comunicación Social.

Piedrahita, J. (2008, 4 de abril). La voz de los recuerdos. El espectador.

Salgado, A. (2005). El presentador de noticias: las cualidades necesarias para una comunicación eficaz. En: Comunicar. No. 25. En línea: http://www.revistacomunicar. com/verpdf.php?numero=25\&articulo $=25-2005-101$

Vargas, N. (2009). El Mañanero y La Cama ¿La voz de los jóvenes? Análisis de los programas matutinos de las emisoras juveniles. Tesis de pregrado. Bogotá: Pontificia Universidad Javeriana -Facultad de Comunicación Y Lenguaje. 